\title{
El rendimiento en la enseñanza a distancia
}

\author{
JAIME SARRAMONA \\ Universidad Autónoma de Barcelona
}

\begin{abstract}
SUMMARY.-The performance use to be a critic theme in distance learning, although there is data enough to judge it objectively. There are performances inherents to the system, as the habit of autotraining or the discipline to study, with the knowledge and the possibility to incorporate habilities. Only the objectives with social aspects may remaine out of the normal achivements.

The discance training, with aplication in the labor world developes an institutional culture of autotraining, which facilitates retraining and permanent education.

We can not forget the consequences on the autors -trainers and tutors-, especialy in relation to the organizational habits and the strict planification of the educational process.
\end{abstract}

\section{1. ¿QUÉ SIGNIFICA RENDIMIENTO EN ENSEÑANZA?}

Hablar de «rendimiento» en la enseñanza resulta tan ambiguo como referirse al «fracaso escolar», término habitualmente contrapuesto al anterior. Sin embargo, será preciso otorgarle algún significado, para luego poder vincularlo con el segundo referente del título de este trabajo, la enseñanza a distancia.

Es evidente que el rendimiento en la enseñanza hace referencia a los resultados obtenidos en un proceso de acción docente $\mathrm{y}$, en un sentido más amplio, en un proceso educativo. El problema reside en determinar qué resultados han de ser considerados en la valoración de la docencia y, sobre todo, cuáles son consecuencia directa de la acción pedagógica previamente planificada. Por consiguiente, bueno será partir de los principios básicos siguientes:

a) En el proceso educativo se obtienen más resultados que los previamente considerados como objetivos a lograr.

b) Los resultados educativos son tanto la consecuencia de la planificación previamente realizada cuanto del proceso aplicativo.

c) En la acción educativa existen siempre un conjunto de variables, difíciles de controlar, que hacen imposible determinar los resultados de manera exacta.

d) Todo programa o acción educativa en que se da participación directa del docente, las variables personales de éste se erigen en factor de diferenciación específica.

Sentados estos principios, por demás comunes en la teoría pedagógica actual, se puede encarar el análisis del rendimiento en la educación, presencial o a distancia, desde una perspectiva estrictamente pedagógica. Es decir, desde una perspectiva que no se limita a la descripción de los hechos sino que propone normas de actuación para modificar 
la realidad, más si ésta no resulta satisfactoria. Este será precisamente el enfoque aquí adoptado.

\section{EL RENDIMIENTO EN LA ENSEÑANZA A DISTANCIA}

El rendimiento suele ser el tema más polémico de la enseñanza a distancia (e.d.), siempre que se analizan sus limitaciones. Aunque son innumerables los estudios realizados para demostrar la eficacia de la e.d. en comparación con la educación presencial, no resulta posible llegar a conclusiones generalizables. La explicación hay que buscarla en los principios enunciados anteriormente, y en las limitaciones intrínsecas de las investigaciones realizadas, que no pueden superar el nivel ideográfico.

Porque los estudios sobre el rendimiento de los programas de e.d. se mueven en el marco de la «evaluación de programas»o, si se prefiere, de la «investigación evaluativa», por lo cual no pueden decir más que lo acontecido en aquel programa concreto. Es así que no debe extrañar la diversidad de variables independientes que resultan significativas.

Una buena recopilación de estudios de tipo causal sobre las variables que afectan al rendimiento académico de la e.d. lo ha realizado Chacón ${ }^{1}$, quien las clasifica en variables relativas a los medios de instrucción, al estudiante y al apoyo institucional. Renuncio a presentar la relación completa de tales variables, que el mencionado autor vincula a investigaciones concretas donde se han mostrado especialmente relevantes; sólo citaré las que el mismo Chacón identificó en su estudio sobre la Universidad Nacional Abierta de Venezuela:

- La cantidad de alumnos inscritos en un curso, así como la dificultad del mismo medida en carga de trabajo, resultan factores negativos para el rendimiento escolar.

- Las estrategias de asesoría, entendidas como acciones realizadas por los tutores para auxiliar al alumno, resultan positivas para los alumnos con menor preparación.

- El conjunto de elementos que se engloban bajo la denominación «diseño instruccional», donde cobra un relieve especial la evaluación, no tienen una incidencia directa sobre la variable rendimiento.

Insisto que estos resultados no son más que otro ejemplo a añadir en la serie de estudios que se podrían citar, en este caso condicionados por el modelo pedagógico de la Universidad Nacional Abierta de Venezuela y el conjunto de prerrequisitos que determinan a su población estudiantil.

Recuperando los principios inicialmente presentados para perfilar el concepto de rendimiento escolar, volveremos los ojos hacia la naturaleza misma de ese rendimiento y su vinculación con la e.d.

\section{RENDIMIENTOS ESPECÍFICOS DE LA ENSEÑANZA A DISTANCIA}

Así como en una metodología presencial convencional se consiguen resultados que son fruto directo de la situación de convivencia en el aula y fuera de ella, del grupo de

1. Factores del rendimiento en los cursos a distancia: aplicación al análisis de vías, UNA, Caracas, 1989. 
alumnos: relaciones interpersonales, conciencia de grupo, socialización, etc., en la e.d. se provocan también resultados que son consecuencia directa de sus características metodológicas.

3.1. En primer término no podemos olvidar lo que se considerarían aprendizajes académicos estrictos. Es evidente que una parte más o menos grande, según los casos, supera las pruebas académicas que establecen los respectivos programas, lo cual demuestra que logran los conocimientos y habilidades establecidos para tal superación. Es aquí donde podríamos entrar en un baile de cifras, porque encontraríamos cursos o programas donde la superación de las pruebas establecidas lo logran la mayoría de los estudiantes, y otros donde sólo lo consiguen una ínfima minoría ${ }^{2}$. Tan sólo una consideración cabría hacer al respecto: si la metodología a distancia no tuviera potencialidad docente y formativa nunca habría un elevado número de alumnos que obtuvieran resultados positivos. Por no seguir en el ámbito formal, donde intervienen muchos elementos difícilmente controlables, tanto en lo que se refiere a las instituciones como a los destinatarios, haré mención a dos programas de enseñanza a distancia en los cuales he participado personalmente como asesor pedagógico de los materiales.

- En un programa elaborado por la Compañía Telefónica Nacional de España en 1986-87, para la reconversión profesional de sus operarios menor calificados en técnicos auxiliares, se diseñó un curso a distancia que preparaba para la superación de una prueba de selección previa a la citada reconversión. El programa fue seguido por unos 3.000 trabajadores, mientras que casi el doble se formaban por otras vías, mayoritariamente cursos presenciales donde se emplearon como textos los materiales del curso a distancia. Aunque los inscritos en la metodología a distancia fueron escogidos por la empresa siguiendo criterios de antigüedad -por tanto eran los trabajadores de más edad y menos hábitos de estudio-, consiguieron el $51 \%$ de las plazas en las pruebas de selección ${ }^{3}$.

- La Caja de Ahorros de Sabadell (Barcelona) tiene establecido un sistema de oposiciones internas para la promoción de una parte de su personal. Como preparación para superar las pruebas de la oposición, hasta 1988 la entidad ofreció formación presencial a los empleados que la deseaban. A partir de esa fecha, la preparación se realiza exclusivamente mediante materiales autoinstructivos y asistencia tutorial a distancia. Las consecuencias han sido una elevación general en todas las puntuaciones medias de las

2. Existe gran dificultad en conocer públicamente las cifras de abandono y de éxito real en las instituciones a distancia, tanto públicas como privadas. Por otra parte, se suele establecer una diferenciación entre los estudiantes que no llegan a comenzar siquiera el proceso (no realizan pruebas de evaluación a distancia ni se han presentado nunca a las presenciales) y quienes abandonan tras haber sido evaluados en alguna ocasión. García Aretio, L. (Rendimiento y abandono en la educación superior a distancia, Uned, Madrid, 1987) cita un abandono en la UNED del 59\% en el primer caso -entre 1973 y 1980 - y del $14 \%$ en el segundo, siendo un $1 \%$ la proporción conjunta de licenciados (p. 308). La UNED de Costa Rica presenta un porcentaje de deserción del 33.9\% en 1989 sobre el total de la matrícula efectiva (Anuario estadístico 1989, UNED, San José). La Open University británica, por su parte, sólo considera alumnos propios de su institución a quienes se inscriben definitivamente tras los tres primeros meses, considerados de inscripción provisional, para así disminuir las cifras estadísticas de deserción.

3. Sarramona, J. (1987), «Experiencia de un programa de formación a distancia para la reconversión profesional en la Compañía Telefónica», XVI Congreso Mundial de la «International Federation of Training and Development Organisations», Madrid. Tales resultados se explican en función de los rendimientos no estrictamente académicos que se comentan más adelante. 
pruebas, lo que demuestra un mayor nivel general en la preparación por parte de los empleados que se presentan a ellas.

Los dos ejemplos aportados pertenecen al ámbito laboral y se inscriben en lo que consideraríamos la formación permanente de adultos, donde nos encontramos con personas motivadas por su promoción y donde la formación se vincula con la actividad laboral. Pero no se olvide que ésta ha sido la gran justificación de la e.d., especialmente en el sector privado, y que ahora empieza a serlo también en el sector público ${ }^{4}$.

Se podrá argumentar que la e.d. resulta factible en la consecución de logros académicos de tipo teórico, pero que los logros de habilidades técnico-manuales, de «saber hacer», requieren de prácticas sólo factibles en laboratorios y talleres que se hallan en los centros de formación presencial. La afirmación resulta bastante evidente, pero sugiere algunas reflexiones:

- Todo programa de e.d. puede y, en ocasiones, debe ser complementado con actividades de tipo presencial, que alcanzarían la consideración de obligatorias cuando se vinculen a aprendizajes que no son fácilmente asequibles fuera de ellas. Otro tanto ocurre con el sistema presencial respecto las sesiones de prácticas fuera de las aulas y, por supuesto, con las exigencias de estudio e investigación personal que también se efectúa fuera de ellas.

- Aunque las habilidades técnico-manuales las he situado en el «saber hacer», bajo este rótulo se incluyen también habilidades de tipo mental, válidas por sí mismas (técnicas de resolución de problemas) o previas a las estrictamente manuales. Es más, no hay actividad técnica que no sea explicada en función de formalizaciones teóricas, de modo que los saberes aplicados se fundamentan en conocimientos científicos (teóricos) y técnicas (normas de proceso) ${ }^{5}$. Estos saberes fundamentales de la actividad práctica son todos accesibles mediante materiales autoformativos, esto es, por metodología a distancia.

- Dentro del campo de las actividades manuales caben aún diversas consideraciones. Muchos de estos conocimientos estrictamente aplicativos son susceptibles de adquisición en el propio domicilio: electrónica, fotografía, dibujo, diseño, etc., si se cuenta con equipos apropiados, tal como se demuestra en multitud de cursos académicos y profesionales. Ello es así por cuanto cada vez más se trabaja con equipos de reducidas dimensiones y los «grandes movimientos corporales» dan paso a tareas de precisión.

- Si se trata de actuar sobre equipos de grandes dimensiones o muy sofisticados, difícilmente se dispone de ellos en los centros académicos. Hace ya tiempo que el sistema educativo formal se ha descolgado de la carrera de la evolución tecnológica en que se halla sumergido el mundo productivo. Solamente una formación vinculada directamente a la actividad laboral puede garantizar el empleo de máquinas y materiales sofisticados, de tecnología punta. La e.d. permite esta vinculación de la formación con la

4. Así ha sido en los países excomunistas de la Europa del Este y en otros casos de la Europa Occidental. Es sintomático, por ejemplo, que el Ministerio de Educación español haya creado en 1992 el Centro para la Innovación y Desarrollo de la Educación a Distancia, donde ya se desarrollan módulos profesionales para ser impartidos en fase experimental, concretamente: mantenimiento en línea, instalador-mantenedor eléctrico, auxiliar de comercio exterior y auxiliar de administración y gestión (Revista de Educación a Distancia, MEC, 5, enero 1993). El Ministerio también ha asignado por concurso público la confección del material necesario para un curso a distancia de formación (FP-3) de jardineras de infancia.

5. Malglaive, G. (1990), Enseigner à des adultes, P.U.F., Paris. 
práctica profesional, al no demandar un contexto académico diferenciado ${ }^{6}$; por ello se está prodigando cada vez más en las empresas.

3.2. Un segundo capítulo de resultados lo constituyen los referentes a los hábitos y actitudes que se vinculan con la manera propia de aprender en la e.d.

En toda la bibliografía referida a las virtudes -también requisitos- de la e.d. se pueden encontrar estas actitudes y hábitos y que se podrían agrupar en los epígrafes siguientes:

- La e.d., puesto que se basa fundamentalmente en el estudio en solitario, desarrolla el hábito del trabajo personal, la disciplina en mantener el estudio durante largos períodos y el espíritu de superación de las dificultades. En síntesis, se desarrolla cuanto se refiere a la independencia personal, creándose el hábito de vencer los problemas sin depender exclusivamente de la ayuda ajena. Porque, si bien el estudiante a distancia puede valerse de la tutoría para resolver las dificultades que le surjan, no suele estar tan fácilmente a su alcance como en la enseñanza presencial (aunque en ésta a veces puede llegar a ser más inaccesible que en la e.d.).

Este fomento de la independencia y del esfuerzo personal, frente a la dependencia que suele fomentar la metodología presencial, es la que puede explicar el éxito de programas como el citado en la Telefónica, donde quienes usaron el mismo material autoformativo en situaciones de docencia presencial a buen seguro confiaron que con sólo «oír» las explicaciones de los profesores tenían suficiente para aprender.

Se podrá añadir que el fomento de los hábitos personales comentados no proporcionan la formación integral, que demandará también de los hábitos de relación y cooperación social. Pero la e.d. no excluye estos últimos, si bien no puede controlar su desarrollo. Muchas actividades a distancia pueden llevarse a cabo en equipo, más si se trata de tareas aplicativas del ámbito profesional; pero el control de resultados deberá ser presencial para tener plenas garantías de adquisición.

Centrados en este mismo apartado, tampoco se puede olvidar que los hábitos estrictos de trabajo personal pueden verse como el reflejo de una sociedad individualista, fomentadora del egoísmo e interés personal por encima de los intereses más propiamente sociales. La e.d. recibirá entonces críticas ideológicas, como supuesta fomentadora de la competitividad, del individualismo a ultranza.

No quisiera extenderme en demostrar la inocencia de la e.d. en el indudable avance de muchos aspectos negativos de la sociedad contemporánea. Se puede recordar que la e.d. tomó un gran desarrollo tanto en los países de democracia liberal como en los de ideología comunista, que la metodología a distancia ha sido democratizadora de la cultura, que el aprendizaje como tal es siempre una actividad personal (aunque se aprenda «al lado de otro»), que la e.d., en definitiva, es sólo una de las posibilidades metodológicas de aprender que se ofrecen a los sujetos.

Aunque no sea esgrimiendo exactamente los argumentos citados, la e.d. puede tener la oposición de personas y colectividades que prefieren el mantenimiento de la enseñanza presencial, porque permite actuar directamente sobre los grupos, con finalidades distintas a las estrictamente académicas. Así, por ejemplo, pueden oponerse grupos sindicales a que se instale la e.d. en las empresas -ocurrió inicialmente en el caso citado

6. Tanto es así que muy bien se puede afirmar que la auténtica educación «a distancia» es aquélla que se realiza en lugares alejados, distantes, de un ámbito de aplicación, aunque esta formación se lleve a cabo por metodología presencial. 
de la Telefónica-, porque les priva de la oportunidad de actuar sobre los grupos aprovechando los cursos de formación presencial. Por contra, razones de tipo político, pueden haber motivado que algunos gobiernos fomentaran la metodología a distancia, secundaria y universitaria, para disminuir las oportunidades de protesta estudiantil.

- Los hábitos comentados tienen también sus consecuencias institucionales cuando los programas a distancia se llevan a cabo en el contexto de una empresa u organismo semejante. Porque se instaura lo que podríamos catalogar como «cultura de la autoformación», lo que se traduce en centrar las iniciativas en los propios sujetos que se forman y no en los responsables directos de la formación. Cultura que luego impregna todos los ámbitos de la institución, estimulando el dinamismo empresarial, gracias a los hábitos de iniciativa arraigados en los trabajadores.

Dentro de estas repercusiones institucionales no se pueden olvidar las que afectan a la organización de la formación. Las empresas optimizan los recursos invertidos en formación y pueden atenderla mediante estructuras muy flexibles, a través de tutorías telefónicas o mediante recursos telemáticos que atienden sus mismos empleados. La atención al unísono y con idénticos contenidos de una amplia masa de empleados permite resolver en breve tiempo problemas de formación, con garantías de equivalencia para todos. Considérese, además, el valor añadido que para la empresa u organismo supone contar con unos materiales de formación, cuya utilidad y proyección siempre van más allá del curso concreto para el que fueron diseñados.

3.3. Este último capítulo, el de los materiales de e.d., abre el interesante análisis de las repercusiones sobre los docentes o autores de los mismos, y que también se pueden catalogar como «resultados académicos»o, si se prefiere, se pueden incluir en la cultura institucional ya comentada.

Las repercusiones a que me refiero merecen una atención especial cuando la e.d. se instaura en una institución -académica o no- de tradición académica presencial. Porque frente a la posibilidad de improvisación que permite la docencia presencial, la metodología a distancia exige siempre planificación y control, por el solo hecho de depender de unos materiales pedagógicos preelaborados al proceso mismo de enseñanza-aprendizaje. Tanto es así que ha sido la e. a d. el auténtico lugar de prueba y de puesta en práctica de los principios tecnológicos más exigentes de la planificación educativa (Gagné, por ejemplo). No se trata ahora de defender un modelo concreto de planificación, ni mucho menos los modelos estrictamente conductistas que imperaron en los años sesenta y setenta (los cuales también eran propuestos en la docencia presencial), sino advertir que la naturaleza de la e.d. favorece - mejor habría que decir, obliga- a unos niveles de rigor que no suelen ser habituales en la metodología presencial.

Sería fácil aportar ejemplos de docentes que rechazan participar en programas de e.d., confeccionando los materiales correspondientes. Y ello porque exigen un esfuerzo personal y un sometimiento a la valoración social del producto, que no tiene parangón con la docencia de aula, donde es la palabra del docente la principal vía de enseñanza y, como suele decirse, «verba volant».

La combinación de docencia presencial y a distancia en un mismo profesor tiene efectos positivos para ambas. De la docencia presencial se puede transferir el valor del diálogo, la atención personalizada de los alumnos y la capacidad de reacción ante situaciones imprevistas. De la docencia a distancia se adquirirá el rigor en la planificación, el ajuste a las variables temporales, el control de todo el proceso. Cuando tales transferen- 
cias se producen en el interior de una institución se erigen en fuente de progreso pedagógico. Estos efectos también se pueden inscribir en el haber de los resultados académicos de la e.d.

Bien cierto que no todos los materiales de la e.d. obedecen a una planificación rigurosa, ni responden a un modelo pedagógico explícito. Aunque no es lo más común, encontraríamos instituciones que se limitan a adoptar una cierta estructura formal para sus materiales, pero dejando libertad a los autores para la concreción de los mismos, sin exigirles más criterios pedagógicos que sus propias opiniones. Pero repito que no es lo más habitual.

En cualquier caso, los efectos o rendimientos académicos de la e.d. que se han indicado requieren de las condiciones oportunas para que se produzcan. La identificación de tales condiciones será el objeto de la segunda parte del trabajo.

\section{CONDICIONES PARA EL LOGRO DE LOS RENDIMIENTOS ACADÉMICOS EN LA ENSEÑAN- ZA A DISTANCIA}

El logro por parte del estudiante a distancia de los objetivos académicos propuestos en el programa demanda de las pertinentes informaciones, sugerencias, orientaciones y controles de evaluación. En este sentido no existe diferencia entre la metodología a distancia y la del aula, con excepción del papel relevante que en la primera toman los materiales informativos, depositarios de la totalidad, o casi, de los elementos citados. Por consiguiente, la dependencia que la e.d. tiene de sus materiales es enorme; cualquier deficiencia en ellos resulta difícil de subsanar, y a veces se detecta cuando ya es demasiado tarde para corregirla.

La incorporación de los más modernos y efectivos conocimientos sobre el aprendizaje, la garantía de que los autores de los materiales están pedagógicamente preparados para su realización, la evaluación previa de los mismos antes de su aplicación definitiva, son otras tantas condiciones básicas que han de exigirse a todo programa serio de e.d.

A título personal, considero adecuado emplear para la planificación de los materiales un modelo tecnológico que incorpore principios cognoscitivistas del aprendizaje, especialmente los que se refieren al logro de estructuras significativas (Bruner), la resolución de problemas (Chadwick) ${ }^{7}$, el «aprender a aprender» (Novak), etc., bajo una estructura de diagnóstico previo y de atención posterior a la dinámica del proceso, tal como se indica en la figura adjunta ${ }^{8}$. De manera general, se deberán tener bien presentes los requisitos siguientes:

a) Atender a necesidades claras de educación, sean explicitadas por los propios destinatarios, por el sector productivo, por las necesidades culturales de la sociedad, etc.

b) Considerar el contexto, tanto institucional como sociocomunitario, al que se destina el curso. Ello implica analizar las tradiciones y hábitos existentes, con el fin de poder «contextualizar» adecuadamente el curso.

7. Chadwick, C. (1988), «Estrategias cognoscitivas y afectivas de aprendizaje», Revista Latinoamericana de Psicología, vol. 20, 2, pp. 163-205.

8. Sarramona, J. «Diseño tecnológico de la enseñanza a distancia», Proyecto PATED, ANCED, Madrid (en prensa). 
c) El conocimiento de las «conductas de entrada» de los destinatarios, entendidas en sentido amplio (conocimientos, actitudes, opiniones, etc.) permitirá ajustar el nivel de los conocimientos a las posibilidades de los receptores, sin excluir la posibilidad de introducir información complementaria para que alcancen el nivel requerido quienes aún no lo poseen.

d) La valoración de los recursos disponibles impondrá la necesaria dosis de realismo en la selección de los medios, tanto en lo que concierne a la actividad docente como discente.

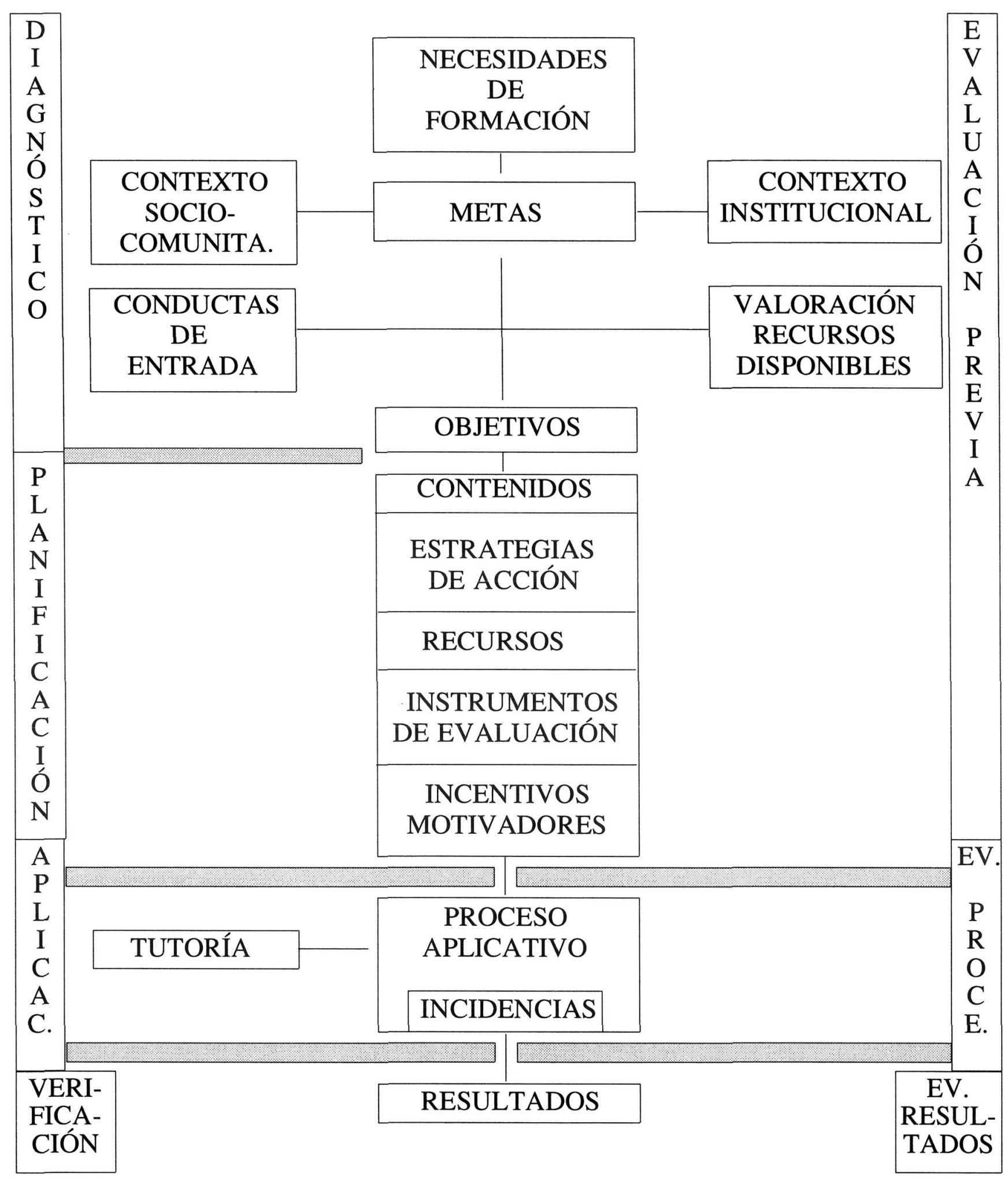

Figura 1. Esquema de diseño tecnológico de un curso a distancia, (Sarramona, 1993). 
e) Los elementos básicos de la planificación: objetivos, contenidos, estrategias de acción, recursos, instrumentos de evaluación e incentivos motivadores, han de seleccionarse en función de sus relaciones mutuas, con el fin de hacer coherente todo el proceso educativo.

f) La puesta en práctica del curso se llevará a cabo a través de la acción tutorial, que controle el proceso y resuelva las posibles incidencias.

Todos estos elementos son básicos para garantizar técnicamente la confección de materiales en la e.d. Cualquier disfunción en uno de ellos puede llevar al fracaso. No será necesario insistir, puesto que el problema es común a cualquier metodología. Sin embargo, dado que la motivación personal aparece siempre como un punto crítico en los estudios sobre el mantenimiento del alumnado en la e.d., bueno será entrar en ello con un poco más de detalle.

Siempre que la elige voluntariamente, cabe suponer en el estudiante a distancia una motivación inicial positiva hacia esta metodología. De no ser así, será preciso despertarla a través de informaciones previas, a poder ser en sesiones cara a cara, pero también a través de la misma estructura y contenidos del programa. Cuando la motivación inicial ya exista sólo habrá que velar por su mantenimiento.

La adecuación del programa a las necesidades de los destinatarios constituye la primera garantía de motivación. Pero al confeccionarlo se podrán incluir incentivos motivadores específicos, tales como:

- Presentación atractiva de las metas y objetivos que se pretenden, poniendo énfasis en la utilidad que el programa representa para los destinatarios.

- Aportación de ejemplos y aplicaciones próximas a los destinatarios.

- Empleo de un lenguaje claro y personalizado.

- Incorporación de orientaciones para el aprendizaje, anticipación de posibles dificultades y estrategias para su solución.

- Incorporación de ilustraciones, color, diagramación, etc., que haga atractivo el estudio de los materiales.

- Inclusión de reforzadores específicos, tales como esquemas, resúmenes, destaques tipográficos, etc.

Puesto que el mantenimiento de la motivación no depende solamente del diseño del curso, sino de su aplicación, habrá que prever también un conjunto de acciones para llevar a cabo durante el proceso aplicativo, aparte de la atención a las incidencias no previsibles que se produzcan. Entre estas acciones de motivación se pueden citar:

- Envío de mensajes de ánimo, cuando surjan fracasos o se produzcan abandonos.

- Envío de mensajes de refuerzo, cuando se superen ciertas fases del curso.

- Demandas de participación en la evaluación del curso, a través de cuestionarios, entrevistas, etc.

- Atención oportuna a las preguntas y cuestiones formuladas por los estudiantes.

Esta última acción abre la perspectiva de todo el conjunto de la atención tutorial. Poco habrá que insistir para destacar la importancia del tutor en la e.d., responsable del proceso pedagógico una vez el material ha sido elaborado y distribuido al estudiante. El tutor es quien evalúa, aclara dudas, anima a los estudiantes a través de la comunicación escrita, telefónica o presencial. Su figura se asemeja a la del profesor de aula, con exclusión hecha de la relevancia que muchas veces toma éste en la transmisión de los conocimientos. El tutor es quien puede vencer la sensación de soledad que suele invadir al 
estudiante a distancia o, si se quiere decir de otro modo, es quien puede evitar el fracaso o abandono del estudiante.

Sería ahora prolijo relatar en detalle todo el cúmulo de tareas que se asignan al tutor $^{9}$, pero, cuando menos, convendría insistir en las siguientes:

- Corrección de los errores que cometan los estudiantes en sus actividades y evaluaciones (al margen de las calificaciones que otorgue) para proporcionarles «feedback» inmediato.

- Proporcionar orientaciones sobre el aprendizaje: advirtiendo lagunas, dando indicaciones de como subsanarlas, facilitando la profundización de los conocimientos si el estudiante lo demanda, ofreciendo ejemplos contextualizados, etc.

- Aclarar cuantas consultas puedan plantearle los alumnos referentes al curso.

- Proporcionar palabras de aliento ante posibles situaciones de desánimo y reforzar a quienes obtienen resultados positivos.

- Interesarse, con discreción, por las situaciones personales que puedan tener incidencia directa en el estudio.

Además de los factores institucionales y pedagógicos que se vinculen a un curso a distancia, no se pueden olvidar los factores sociales que envuelven al estudiante. Factores que van desde el reconocimiento profesional de la formación obtenida hasta la misma valoración social que merezca la metodología a distancia. Y es indudable que en este último capítulo aún queda mucha labor por hacer, porque la e.d. se enfrenta a menudo con un cierto menosprecio social que es la resultante del desconocimiento de su funcionamiento y posibilidades. A sabiendas que el sistema educativo presencial suele ser el más reacio a aceptar otras posibilidades de enseñanza que no sean las directamente gestionadas por los docentes en el aula.

Todo programa de e.d., por tanto, ha de contemplar la necesidad de ofrecer una imagen de prestigio, de seriedad, que aleje los prejuicios y fomente la satisfacción de participar en él. Tal vez en un futuro estos requisitos no sean ya necesarios, pero hoy aún lo es.

A medio camino entre las variables psicológicas y sociales está otro de los puntos críticos que la e.d. ha de atender; me refiero a la sensación de soledad que suele invadir al estudiante. Cuanto mayor ha sido la dependencia engendrada en la educación presencial, mayor dificultad supone el estudio en solitario, el determinar por uno mismo el ritmo de aprendizaje, el exigirse a sí mismo sin que se reciba la presión constante del grupo-clase o del docente.

Hasta que los estudiantes no arraiguen los hábitos de estudio en solitario, los programas a distancia habrán de procurar crear un sentimiento de colectividad, mediante comunicaciones periódicas, programas específicos de radio, revistas, centros de reunión y de atención presencial, etc. Todo ello complementado con la demanda de ciertas actividades para ser realizadas en grupo, siempre que sea posible. Si el trabajo en equipo no es fácilmente realizable puede serlo la visita a centros, la entrevista a personas, la asistencia a actos académicos o culturales, que también desarrollan la sensación de pertenencia institucional.

9. La Asociación de Escuelas Europeas por Correspondencia (EHSC) ha elaborado un Curso para tutores donde aparecen todas las tareas que corresponden al tutor y la manera de llevarlas a cabo. 
EN DEFINITIVA...

La e.d. es una metodología general de educación donde lo sustantivo está en el término «enseñanza» o, si se prefiere, «educación», porque el concepto de «distancia» es susceptible de muchas interpretaciones. Sin embargo un elemento básico la caracteriza y se erige en justificación de sus principales ventajas y limitaciones, la necesidad de anticipar, a través de la planificación y materiales pertinentes, el proceso de enseñanzaaprendizaje.

Los efectos logrados dependen, pues, básicamente de la planificación, lo cual otorga a la e.d. un carácter de metodología tecnológica, donde la improvisación no puede ser más que la respuesta a situaciones claramente imprevisibles, nunca una estrategia de acción constante. Esto por lo que se refiere al ámbito docente. Por parte del discente, las exigencias de madurez, dedicación e interés aparecen como fundamentales.

En último extremo, algo en común tienen la educación a distancia y la presencial: que siempre hay que hablar de un «hic et nunc», porque cada caso tiene su propia idiosincrasia. 